\title{
Modelos de predição aplicados a cinética de secagem solar da goiaba
}

\author{
Prediction models applied to guava solar drying kinetics \\ Modelos de predicción aplicados a la cinética del secado solar de la guayaba
}

Recebido: 23/08/2021 | Revisado: 03/09/2021 | Aceito: 06/09/2021 | Publicado: 07/09/2021

\author{
Bárbara Valéria Pereira Lins \\ ORCID: https://orcid.org/0000-0002-0331-1096 \\ Universidade Federal de Campina Grande, Brasil \\ E-mail: barbaravplins@gmail.com \\ Fabiana Pimentel Macêdo Farias \\ ORCID: https://orcid.org/0000-0003-4168-9601 \\ Universidade Federal de Campina Grande, Brasil \\ E-mail: fabiana.pimentel@professor.ufcg.edu.br \\ Débora Rafaelly Soares Silva \\ ORCID: https://orcid.org/0000-0002-7727-9535 \\ Universidade Federal de Campina Grande, Brasil \\ E-mail: deborarafaelly@yahoo.com.br \\ Morgana Fabílola Cunha Silva Canuto \\ ORCID: https://orcid.org/0000-0003-4880-3788 \\ Universidade Federal de Campina Grande, Brasil \\ E-mail: morganafabiola@ hotmail.com \\ Ana Clara Sampaio Meira \\ ORCID: https://orcid.org/0000-0002-2912-4803 \\ Universidade Federal de Campina Grande, Brasil \\ E-mail: ana-clarasm@hotmail.com
}

\begin{abstract}
Resumo
A goiaba apresenta excelentes características nutricionais, sendo considerada uma importante matéria prima na indústria de alimentos. Para a utilização eficiente desse fruto, é necessária a adoção de técnicas de conservação que retardem as perdas de qualidade decorrentes do processo de amadurecimento. A secagem por meio de energia solar devido ao seu potencial e baixo custo, configura-se como uma alternativa economicamente viável para a conservação de produtos agroindustriais. Assim, objetivou-se com esse estudo analisar o processo de desidratação da goiaba e descrever a cinética de secagem utilizando secador solar, bem como ajustar os dados experimentais a modelagem matemática. Foram utilizadas goiabas da variedade Paluma no estádio de maturação 3 (verde-amarelo), adquiridas no comércio local. As amostras foram acondicionadas em cestas de arame e submetidas à secagem em monocamada em triplicata, através de um secador solar. Os dados experimentais de secagem da goiaba foram ajustados às equações de Page, Henderson e Pabis, Lewis, Cavalcanti Mata e Thompson. O secador solar promoveu a obtenção de resultados satisfatórios no processo de secagem da goiaba, onde a melhor predição ao processo de cinética de secagem foi obtida pelo modelo de Page.
\end{abstract}

Palavras-chave: Psidium guajava L.; Desidratação; Pós-colheita.

\begin{abstract}
Guava has excellent nutritional characteristics, being considered an important raw material in the food industry. For the efficient use of this fruit, it is necessary to adopt conservation techniques that delay the loss of quality resulting from the ripening process. Drying using solar energy, due to its potential and low cost, is an economically viable alternative for the conservation of agro-industrial products. Thus, the objective of this study was to analyze the guava dehydration process and describe the drying kinetics using a solar dryer, as well as adjust the experimental data to mathematical modeling. Guavas of the Paluma variety were used at ripening stage 3 (green-yellow), purchased from local stores. The samples were placed in wire baskets and subjected to drying in a single layer in triplicate, using a solar dryer. The guava drying experimental data were fitted to the equations of Page, Henderson and Pabis, Lewis, Cavalcanti Mata and Thompson. The solar dryer provided satisfactory results in the guava drying process, where the best prediction of the drying kinetics process was obtained by the Page model.
\end{abstract}

Keywords: Psidium guajava L.; Dehydration; Post-harvest.

\section{Resumen}

La guayaba tiene excelentes características nutricionales, siendo considerada una materia prima importante en la industria alimentaria. Para el uso eficiente de esta fruta, es necesario adoptar técnicas de conservación que retrasen la pérdida de calidad resultante del proceso de maduración. El secado mediante energía solar, por su potencial y bajo costo, es una alternativa económicamente viable para la conservación de productos agroindustriales. Así, el objetivo 
de este estudio fue analizar el proceso de deshidratación de la guayaba y describir la cinética de secado utilizando un secador solar, así como ajustar los datos experimentales a modelos matemáticos. Se utilizaron guayabas de la variedad Paluma en la etapa de maduración 3 (verde-amarillo), compradas en las tiendas locales. Las muestras se colocaron en cestas de alambre y se secaron en una sola capa por triplicado, utilizando un secador solar. Los datos experimentales de secado de guayaba se ajustaron a las ecuaciones de Page, Henderson y Pabis, Lewis, Cavalcanti Mata y Thompson. El secador solar proporcionó resultados satisfactorios en el proceso de secado de la guayaba, donde la mejor predicción de la cinética del proceso de secado se obtuvo mediante el modelo de Page.

Palabras clave: Psidium guajava L.; Deshidración; Poscosecha.

\section{Introdução}

Um grande problema enfrentado pelos fruticultores é a conservação dos frutos maduros, pois grande parte da colheita é desperdiçada. Uma das principais causas de perdas é a deterioração, devido à alta quantidade de água livre presente nos frutos. De acordo com (Galli et al., 2015), a goiaba (Psidium guajava L.) é uma espécie bastante importante nas regiões tropicais e subtropicais se destacando por sua excelente qualidade nutricional pelo seu alto valor nutritivo (rico em vitaminas A, C, ferro, cálcio e fósforo), e pelas propriedades sensoriais, alto rendimento por área e polpa com elevada qualidade industrial (doces, geleias e sucos), além de possuir alta aceitação do mercado consumidor por ser uma fruta altamente saborosa. Entretanto, devido ao intenso metabolismo durante o amadurecimento, esses frutos senescem rapidamente, impedindo o armazenamento por períodos prolongados (Vila et al., 2007).

A indústria de alimentos busca constantemente, inovações que possam favorecer o aproveitamento e o aumento do nicho de mercado para alimentos relativamente conhecidos, como é o caso da polpa de goiaba. Além disso, procura-se desenvolver métodos que possam conservar alimentos por um período de tempo maior, mantendo da melhor maneira possível suas características sensoriais e nutricionais, visto que, para levar os frutos a outras localidades, de maneira segura para o consumo, é necessário o emprego de tecnologias adequadas.

A secagem consiste de um processo de transferência de calor e massa entre o produto e o meio utilizado para secá-lo, geralmente o ar. Durante a secagem, a retirada da umidade é obtida pela movimentação das moléculas de água, decorrente de uma diferença de pressão de vapor d'água entre a superfície do produto a ser secado e o ar que o envolve (Silva, 2000). Dentre as diversas técnicas de secagem destaca-se a secagem solar, a qual utiliza a energia solar, fonte de energia sustentável para a secagem de produtos da agroindústria. Apresentando-se dessa forma como uma alternativa economicamente viável, devido seu potencial e baixo custo. A secagem por meio de energia solar é realizada em secadores solares, que podem ser de dois tipos: secador de exposição direta e secador de exposição indireta, podendo haver convecção natural ou forçada.

As informações presentes nas curvas de secagem são de grande importância para o desenvolvimento dos processos e para o dimensionamento de equipamentos, através delas, pode-se estimar o tempo da secagem de uma quantidade de produtos e, com o tempo necessário para a produção, tem-se a estimativa do gasto energético que refletirá no custo de processamento e, consequentemente, terá influência no preço final do produto (Vilela \& Artur, 2008).

O ajuste dos dados experimentais a modelos matemáticos proporciona melhor controle sobre o processo, além de poder ser utilizado como uma forma de avaliar novas práticas de secagem (Wang et al., 2018).

Neste contexto, o presente trabalho teve como objetivo analisar o processo de desidratação da goiaba e descrever a cinética de secagem utilizando secador solar, bem como ajustar os dados experimentais a modelagem matemática.

\section{Metodologia}

O trabalho foi desenvolvido no Laboratório de Hidráulica e Fenômenos de Transporte da Unidade Acadêmica de Tecnologia do Desenvolvimento (UATEC), do Centro de Desenvolvimento Sustentável do Semiárido (CDSA), da Universidade Federal de Campina Grande (UFCG), em Sumé, Paraíba. A pesquisa científica realizada foi de caráter 
quantitativo, conforme descrito por Pereira et. al (2018) caracteriza-se por gerar conjuntos ou massas de dados que podem ser analisados por meio de técnicas matemáticas. Foram utilizadas goiabas da variedade Paluma no estádio de maturação 3 (verdeamarelo), adquiridas no comércio local. Os frutos foram lavados em água corrente; em seguida, sanitizados em uma solução de $50 \mathrm{ppm}$ de cloro livre, durante cinco minutos, e enxaguados em água destilada; logo após, as goiabas foram colocadas para secar em bancadas sobre papel toalha. Após a sanitização, os frutos foram descascados, e cortados transversalmente em fatias de aproximadamente $1 \mathrm{~cm}$. Em seguida, as amostras foram acondicionadas em cestas de arame e submetidas à secagem em monocamada em triplicata, através de um secador solar.

A secagem ocorreu durante o período de dois dias, no primeiro dia do processo, as amostras foram expostas a radiação solar a partir das 08:00 permanecendo até às 17:00h, após esse horário o secador solar foi recolhido e armazenado no laboratório, para evitar que houvesse ganho de teor de água pelas amostras; no dia seguinte, o secador foi exposto juntamente com as amostras à radiação solar até o término do processo, ou seja, quando as amostras atingiram o peso constante.

Para a determinação da cinética de secagem, a matéria-prima foi avaliada quanto ao teor de água, inicial e final, a partir do método padrão da estufa a $105 \pm 3{ }^{\circ} \mathrm{C}$, por um período de $24 \mathrm{~h}$. A taxa de secagem foi determinada através da aferição da massa das amostras em intervalos regulares durante a secagem, através de uma balança semianalítica digital com precisão de $0,01 \mathrm{~g}$. Os dados obtidos foram utilizados para avaliar a perda de água em função do tempo de secagem, sendo expressos na forma de razão de teor de água (RX) (Eq. 1).

$$
R X=\frac{X-X_{e}}{X_{1}-X_{e}}
$$

Em que: RX - razão de teor de água do produto (adimensional); X - teor de água do produto (b.s.); Xi - teor de água inicial do produto (b.s.); Xe - teor de água de equilíbrio do produto (b.s.).

As equações de Page, Henderson e Pabis, Lewis, Cavalcanti Mata e Thompson, foram utilizadas na predição dos dados experimentais da secagem da goiaba, conforme descrito na Tabela 1.

Tabela 1 - Modelos matemáticos utilizados na predição da secagem da goiaba.

\begin{tabular}{cc}
\hline Modelos & Equação \\
\hline PAGE & $R X=\exp \left(-k \cdot t^{n}\right)$ \\
HENDERSON e PABIS & $\mathrm{RX}=\mathrm{a} \cdot \exp (-\mathrm{k} \cdot \mathrm{t})$ \\
LEWIS & $R X=\exp (-k \cdot t)$ \\
CAVALCANTI MATA & $\mathrm{RX}=\mathrm{a}_{1} \cdot \exp \left(-\mathrm{k}_{1} \cdot \mathrm{t}^{\mathrm{n}_{1}}\right)+\mathrm{a}_{2} \cdot \exp \left(-\mathrm{k}_{1} \cdot \mathrm{t}^{\mathrm{n}_{2}}\right)+\mathrm{a}_{\mathrm{a}}$ \\
THOMPSON & $\mathrm{RX}=\exp \left\{\left[-\mathrm{a}-\left(\mathrm{a}^{2}+4 \cdot \mathrm{b}, \mathrm{t}\right)^{0,5}\right] / 2, \mathrm{~b}\right\}$ \\
\hline
\end{tabular}

Em que: RX - Razão do teor de água; $\mathrm{t}$ - tempo (min); k, k1 - constantes de secagem; n, n1, n2, a1, a2, a3 e b - coeficientes das equações. Fonte: Autores. 
Para a predição dos modelos matemáticos aos dados experimentais da cinética de secagem da goiaba foram realizadas análises de regressão não linear, através do método numérico Quasi-newton contido no programa estatístico Statsoft Statistica 7.0. Como critério de seleção, para expressar o grau de ajuste de cada modelo considerou a magnitude do coeficiente de determinação $\left(\mathrm{R}^{2}\right)$, o desvio quadrático médio (DQM) (Eq. 2) e a verificação da tendência de distribuição dos resíduos (RES). Sendo considerado como ajuste satisfatório o modelo que obtive os maiores valores de R2, os menores valores de DQM e a distribuição aleatória dos resíduos.

$$
D Q M=\sqrt{\frac{\sum\left(R U_{p r e d}-R U_{e x p}\right)^{2}}{N}}
$$

Em que: DQM: desvio quadrático médio; RUpred: razão de umidade predita pelo modelo; RUexp: razão de umidade experimental; N: número de observações realizadas durante o experimento.

\section{Resultados e Discussão}

Na Figura 1, tem-se a representação gráfica do comportamento adimensional da razão do teor de água em função do tempo de secagem da goiaba. O tempo máximo requerido para a secagem solar da goiaba foi de 1080 min, o teor de água inicial da amostra foi de $83,5 \%$ b.u, após a secagem obteve-se o teor final de $8,98 \%$ b.u, de modo, que ocasionou uma redução média de $89,2 \%$ do teor de água inicial do produto avaliado.

Coelho et al. (2019) ao realizarem a secagem da banana cv. terra com uso de energia solar térmica sob exposição direta, também obtiveram o tempo total de secagem de $1080 \mathrm{~min}$ (18 h) para os dois tipos de protótipos utilizados.

A secagem da goiaba ocorreu em taxa decrescente, a razão de teor de água reduziu acentuadamente até o período de $480 \mathrm{mim}$, posteriormente, esta redução ocorreu lentamente à medida em que aumentava o tempo de secagem. Segundo Kashaninejad et al. (2007), este fato ocorre devido a maior resistência a transferência de calor e massa do interior do produto para sua superfície, tornando o processo de secagem mais lento em função do tempo. Sendo este fenômeno, comumente relatado em estudos realizados para avaliar a secagem solar de produtos agrícolas, tais como: manga "Espada" (Coelho et al., 2019), banana "Prata" (Camelo et al., 2019), tomate (Barbosa et al., 2018), manga "Tommy Aktins" (Coelho et al., 2018), coentro (Sousa et al., 2018). 
Figura 1. Curva de secagem solar da goiaba.

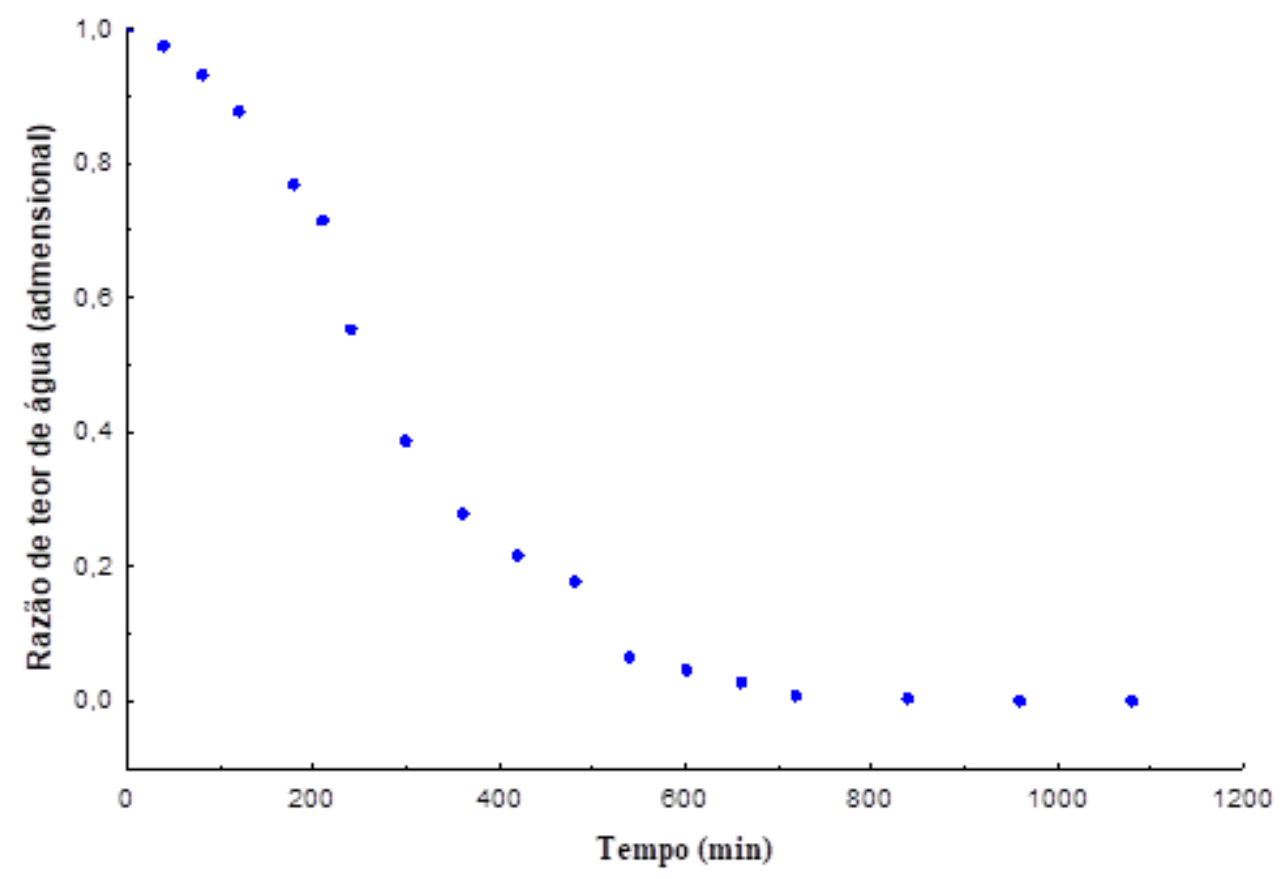

Fonte: Autores.

Na Tabela 2, encontram-se os valores estatísticos dos coeficientes de determinação $\left(R^{2}\right)$ e o desvio quadrático médio (DQM), bem como, os parâmetros dos modelos matemáticos analisados na cinética de secagem da goiaba com uso de secador solar.

O parâmetro "k", de acordo com Goneli et al. (2007), representa o efeito das condições externas de secagem, verificase que o valor máximo deste parâmetro foi obtido para o modelo de Henderson e Pabis. Enquanto, que o menor índice de "k", foi obtido para o modelo de Page. Coelho et al. (2019) ao realizarem a secagem solar da banana cv. Terra, também verificaram que o valor máximo do parâmetro " $k$ " foi obtido para o modelo de Henderson e Pabis.

Com relação ao parâmetro " $n$ ", verifica-se que o modelo de Page obteve valor superior aos obtidos através do modelo de Cavalcanti Mata. Segundo Guedes e Faria (2000), o parâmetro "n" possui um efeito de moderação do tempo e corrige os prováveis erros resultantes de se negligenciar a resistência interna para a transferência de água. Em concordância com este estudo, Mendonça et al. (2015) ao determinarem as curvas de secagem das sementes de andiroba em secador solar, verificaram que este parâmetro foi superior para o modelo de Page quando comparado aos demais modelos avaliados.

Quanto ao parâmetro "a", presente nos modelos de Henderson e Pabis, Cavalcanti Mata e Thompson, verifica-se que contrariamente aos demais modelos analisados, este parâmetro apresentou valor negativo para Thompson.

Observa-se que dentre os modelos aplicados na predição dos dados experimentais, apenas os modelos de Page e Cavalcanti Mata apresentaram coeficientes de determinação $\left(R^{2}\right)$ superiores a 99,0\%, tais resultados indicam uma representação satisfatória dos modelos ao processo de secagem estudado. Contudo, o uso do coeficiente de determinação como único critério não é um bom parâmetro para a seleção de modelos matemáticos não lineares para representar o processo de secagem tornando-se necessária a análise conjunta de outros parâmetros estatísticos (Madamba et al., 1996). Di Domenico e Bavaresco (2018), ao utilizarem o secador solar na desidratação da carambola, também obtiveram o melhor ajuste aos dados experimentais por meio do modelo de Page, cujo coeficiente de determinação foi superior a 98,0\%.

Quanto ao desvio quadrático médio (DQM), verifica-se que os modelos de Page e Cavalcanti Mata, obtiveram os menores valores de $\mathrm{DQM}(\mathrm{DQM}<0,05)$ para secagem estudada. Os valores inferiores de $\mathrm{DQM}$ reportados por estes modelos, 
indicam maior representatividade aos dados experimentais, quando comparados aos demais modelos utilizados neste estudo.

Tabela 2 - Parâmetros dos modelos analisados, coeficientes de determinação $\left(\mathrm{R}^{2}\right)$, desvio quadrático médio (DQM) da cinética de secagem solar da goiaba.

\begin{tabular}{|c|c|c|c|c|c|c|c|c|}
\hline MODELOS & \multicolumn{8}{|c|}{ PARÂMETROS } \\
\hline \multirow{2}{*}{ Page } & \multicolumn{3}{|l|}{$\mathbf{n}$} & \multicolumn{3}{|l|}{$\mathbf{k}$} & $\mathbf{R}^{2}$ & DQM \\
\hline & \multicolumn{3}{|l|}{1,4674} & \multicolumn{3}{|l|}{0,0002} & 99,35 & 0,0461 \\
\hline \multirow{2}{*}{$\begin{array}{l}\text { Henderson } \\
\text { Pabis }\end{array}$} & \multicolumn{3}{|l|}{$\mathbf{a}$} & \multicolumn{3}{|l|}{$\mathbf{k}$} & $\mathbf{R}^{2}$ & DQM \\
\hline & \multicolumn{3}{|l|}{1,1055} & \multicolumn{3}{|l|}{0,0032} & 97,73 & 0,0860 \\
\hline \multirow{2}{*}{ Lewis } & \multicolumn{6}{|l|}{$\mathbf{K}$} & $\mathbf{R}^{2}$ & DQM \\
\hline & \multicolumn{6}{|l|}{0,0028} & 96,64 & 0,1042 \\
\hline \multirow{2}{*}{ Cavalcanti Mata } & $\mathbf{a}_{1}$ & $\mathbf{k}_{1}$ & $\mathbf{n}_{1}$ & $\mathbf{a}_{2}$ & $\mathbf{n}_{2}$ & $\mathbf{a} 3$ & $\mathbf{R}^{2}$ & DQM \\
\hline & 1,1019 & 0,0003 & 1,3789 & $-0,9704$ & 0,5381 & 0,9054 & 99,44 & 0,0427 \\
\hline \multirow{2}{*}{ Thompson } & \multicolumn{3}{|l|}{$\mathbf{a}$} & \multicolumn{3}{|l|}{ b } & $\mathbf{R}^{2}$ & DQM \\
\hline & \multicolumn{3}{|c|}{$-1380,33$} & \multicolumn{3}{|l|}{2,2110} & 97,72 & 0,0861 \\
\hline
\end{tabular}

Fonte: Autores.

Como critério de seleção do melhor modelo matemático de predição aos dados experimentais desse estudo, também foi utilizada a tendência de distribuição de resíduos (Tabela 3). De acordo com Goneli (2008) um modelo é considerado aceitável se a distribuição dos resíduos for aleatória, ou seja, se os valores dos resíduos se encontrarem próximos à faixa horizontal em torno de zero e, caso o modelo apresente distribuição dos resíduos de forma tendenciosa, o mesmo é classificado como inadequado para representar o fenômeno. Apenas os modelos de Page e Cavalcanti Mata representaram a distribuição aleatória dos resíduos, enfatizando os resultados descritos anteriormente pelos parâmetros de seleção adotados nesse estudo, ao demonstrarem que os demais modelos matemáticos avaliados não se adequaram satisfatoriamente a cinética de secagem da goiaba. Mendonça et al. (2015) ao estudarem a cinética de secagem das sementes de andiroba em secador solar, também verificaram que o modelo de Page apresentou distribuição aleatória para as condições de secagem avaliadas.

Tabela 3. Tendência de distribuição de resíduos (RES) para os modelos ajustados aos dados experimentais da cinética de secagem da goiaba.

\begin{tabular}{cc}
\hline MODELO & RES \\
\hline PAGE & ALEATÓRIO \\
HENDERSON \& PABIS & TENDENCIOSO \\
LEWIS & TENDENCIOSO \\
CAVALCANTI MATA & ALEATÓRIO \\
THOMPSON & TENDENCIOSO \\
\hline
\end{tabular}

Fonte: Autores. 
Baseado nos modelos matemáticos testados, pôde-se observar que as melhores predições foram desenvolvidas pelos modelos de Page e Cavalcanti Mata, em razão de terem obtido os maiores coeficientes de determinação $\left(R^{2}=99,44\right.$ e 99,35) e, os menores desvios médios (DQM =0,0427 e 0,0461), respectivamente. Além, da distribuição de resíduos que ocorreu de forma aleatória para ambos os modelos na condição estudada, de modo, que podem ser recomendados e utilizados para a representação do fenômeno de cinética de secagem da goiaba.

Apesar dos modelos de Page e Cavalcanti Mata serem recomendados para representação desse estudo, foi selecionado o modelo de Page para representar a cinética de secagem da goiaba em secador solar. Em virtude desse modelo ser considerado matematicamente mais simples, apresentando o número reduzido de parâmetros, que simplifica o processo de simulação de secagem.

Resultado semelhante foi relatado por Nunes e Pelegrino (2017), ao estudarem a cinética de secagem da banana prata (Musa spp.) utilizando um secador solar, reportaram que os modelos matemáticos de Page e Cavalcanti Mata foram satisfatórios na predição do estudo. Ainda, de acordo com os autores, o modelo de Page obteve uma melhor significância estatística, sendo considerado o melhor modelo para a representação do estudo. Camelo et al. (2019) também verificaram que o modelo Page se ajustou melhor aos dados experimentais para a secagem solar da banana prata (Musa spp.), nas formas cilíndrica e em disco. Santos et al. (2020) ao aplicarem a modelagem matemática a cinética de secagem das cascas de pitomba, constataram que dentre os modelos matemáticos avaliados, Page ajustou-se satisfatoriamente na predição da secagem.

Na Figura 2, tem-se a curva da cinética de secagem da goiaba, representada pelo modelo de Page. Observa-se que a curva da cinética de secagem gerada pela equação de Page apresentou maior concordância com os resultados observados para os intervalos de tempo avaliados, reforçando a aplicabilidade desse modelo na predição da cinética de secagem da goiaba com uso do secador solar.

Figura 2. Curvas da cinética de secagem da goiaba com ajuste dos dados experimentais pela equação matemática de Page, obtidas por regressão não linear.

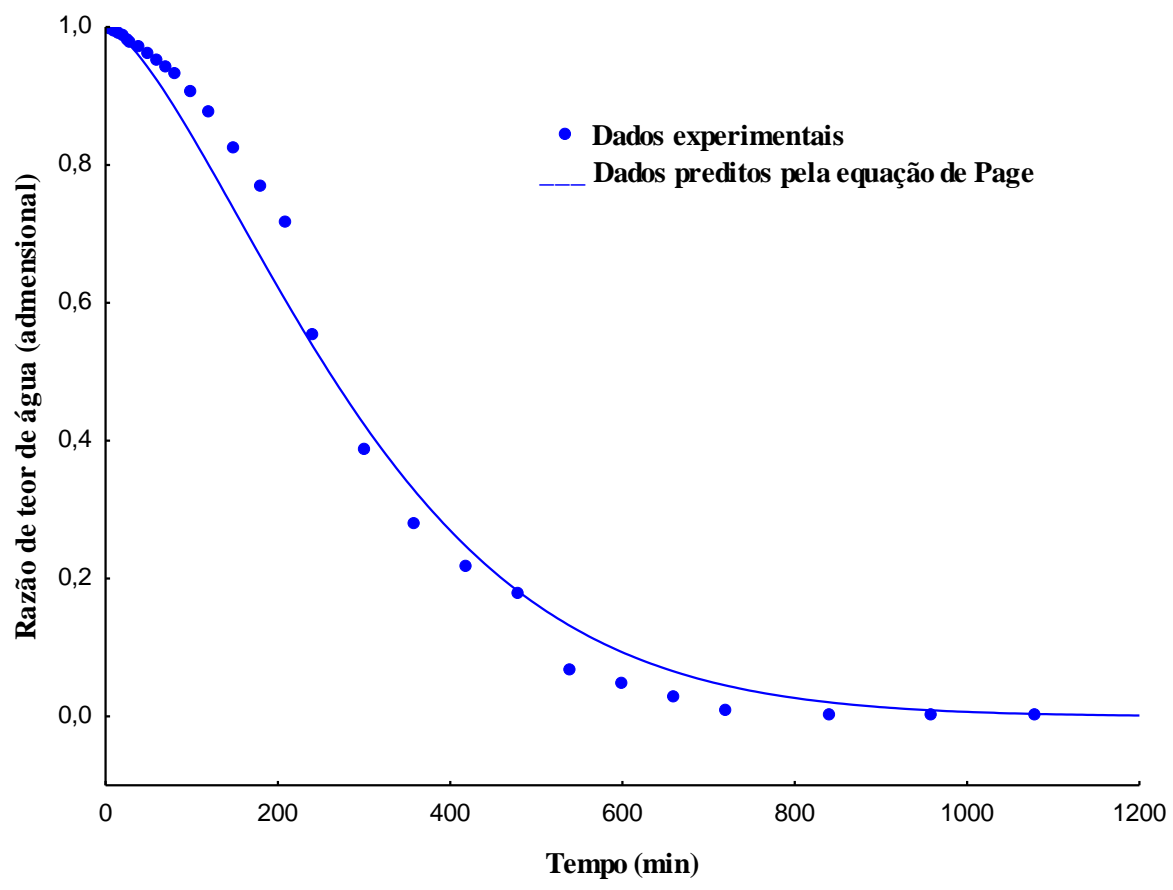

Fonte: Autores. 


\section{Conclusão}

O secador solar promoveu a obtenção de resultados satisfatórios no processo de secagem dos frutos, podendo ser considerado uma alternativa viável na conservação pós-colheita da goiaba.

O modelo matemático proposto por Page apresentou a melhor predição ao processo de cinética de secagem da goiaba, obtendo coeficiente de determinação $\left(\mathrm{R}^{2}\right)$ superior a 99\%, desvio quadrático médio (DQM) inferior a 0,05 e a distribuição de resíduo de forma aleatória, para a condição de secagem avaliada.

Em futuros trabalhos, a sugestão é utilizar o secador solar de exposição direta no processo de desidratação de outros produtos, a fim de obter as cinéticas de secagem e ajustar os dados experimentais a modelagem matemática.

\section{Referências}

Barbosa, E. G., Belém, G. L., \& Danusa, G. C. (2018). Avaliação da secagem do tomate (lycopersicon esculentum mill) em corte transversal em estufa e secador solar. In: Congresso Nacional da Diversidade do Semiárido - CONADIS, Anais - CONADIS, Campina Grande - PB.

Camelo, R. S. S., Paes, J. L., Braz, M. R. S., Bruggianesi, G., \& Guimarães, C. L. (2019). Cinética de secagem da banana prata (Musa spp.) em secador híbrido. Revista Ciência Agronômica, 50 (3), 353-360.

Coelho, B. E. S., Nascimento, M. M., Carvalho, I. R. B., Sousa, K. dos S. M., Machado, N. S., \& Figueiredo Neto, A. (2019). Curva de secagem solar e avaliação físico-química da manga "Espada”. Journal of Environmental Analysis and Progress. 4 (3), 187-194.

Coelho, B. E. S., Sá, C. H. de, Silva, L. F. M. da, Sousa, K. dos S. M. de, \& Machado, N. S. (2019). Ajuste de modelos matemáticos a curva de secagem da banana cv. terra com uso de energia solar térmica sob exposição direta. Revista Craibeiras de Agroecologia, 4 (1), 76-88.

Coelho, B. E. S., Silva, L. F. M., Novaes, M. S. S., Sousa, K. D. S. M. de, \& Machado, N. S. (2018). Cinética de secagem da manga "tommy aktins" em secador solar de baixo custo e com convecção forçada de ar. In: Anais do $5^{\circ}$ Congresso Técnico Científico da Engenharia e da Agronomia - CONTECC, Anais do $5^{\circ}$ Congresso Técnico Científico da Engenharia e da Agronomia, Maceió - AL.

Di Domênico, C. N. B., \& Bavaresco, M. (2018). Utilização de secador solar na desidratação de carambola: estudo experimental e modelagem matemática. Tecnologia \& Ciência Agropecuária, 12, 61-64.

Galli, J. A., Fischer, I. H., Palharini, M. C. de A., \& Michelotto, M. D. (2015). Quantificação de doenças pós-colheita em acessos de goiabeira cultivados em sistema orgânico. Pesquisa Agropecuária Tropical, 45 (2), 225-230.

Gomes, I. A., Medeiros, P. G. P., \& Grilo, B. G. (2018). Secador solar de frutas: Análise de dois sistemas. In: VII Congresso Brasileiro de Energia Solar, Gramado - RS.

Goneli, A. L. D. (2008). Variação das propriedades físico-mecânicas e da qualidade da mamona (Ricinus communis L.) durante a secagem e o armazenamento. Viçosa, Tese (Doutorado em Engenharia Agrícola), Universidade Federal de Viçosa.

Goneli, A. L. D., Corrêa, P. C., Resende, O., \& Reis Neto, S. A. (2007). Estudo da difusão de umidade em grãos de trigo durante a secagem. Ciência $e$ Tecnologia de Alimentos, 27 (1), 135-140.

Guedes, A. M. M., \& Faria, L. J. G. (2000). Determinação da constante de secagem de urucum (Bixa orellana L.) em secador convectivo de leito fixo. Revista Brasileira de Produtos Agroindustriais, 2 (1), 73-86.

Kashaninejad, M., MortazavI, A., Safekordi, A., \& Tabil, L.G. (2007). Thin-layer drying characteristics and modeling of pistachio nuts. Journal of Food Engineering, 78, 98-108.

Madamba, P. S., Driscoll, R. H., \& Buckle, K. A. (1996). The Thin-layer Drying Characteristics of Garlic Slices. Journal of Food Engineering, 29 , 75-97.

Mendonça, A. P., Sampaio, P. de T. B., Almeida, F. de A. C., Ferreira, R. F., \& Novais, J. M. (2015). Determinação das curvas de secagem das sementes de andiroba em secador solar. Revista Brasileira de Engenharia Agrícola e Ambiental, 19 (4), 382-387.

Nunes, A. G., \& Pellegrino, N. S. L. (2017). Tecnologias Sustentáveis: Desenvolvimento de um Secador Solar para Secagem de Frutas. Revista Espacios, 38 (53), p. 9-16.

Pereira, A. S., Shitsuka, D. M., Parreira, F. J., \& Shitsuka, R. (2018). Metodologia da pesquisa científica. UFSM, 119 p.

Santos, N. C., Almeida, R. L. J., Pereira, T. dos S., Queiroga, A. P. R. de, Silva, V. M. de A., Amaral, D. S. do, Almeida, R. D., Ribeiro, V. H. de A., Barros, E. R., \& Silva, L. R. I. da. (2020). Mathematical modeling applied to the drying kinetics of pitomba bark (Talisia esculenta). Research, Society and Development, 9 (2), 921-986.

Silva, J. S., Filho. A. F. L., \& Berbert, P. A. (2000). Secagem e Armazenagem de Produtos Agrícolas. Cap 5, $395-464$.

Sousa, K. dos S. M., Silva, T. B. de S., Lessa, T. B. da S., Barbosa, K. dos S., Pereira-silva, V., \& Machado, N. S. (2018). Estudo da cinética de secagem do coentro sob ação da radiação direta e difusa. Revista Nucleus, 15 (2), 423-432. 
Research, Society and Development, v. 10, n. 11, e519101119761, 2021

(CC BY 4.0) | ISSN 2525-3409 | DOI: http://dx.doi.org/10.33448/rsd-v10i11.19761

Vila, M. T. R., Lima, L. C. O., Vilas Boas, E. V. B., Doll Hojo, E. T., Rodrigues, L. J., \& Paula, N. R. F. (2007). Caracterização química e bioquímica de goiabas armazenadas sob refrigeração e atmosfera modificada. Ciência e Agrotecnologia, 31 (5), 1435-1442.

Vilela, C. A. A., \& Artur, P. O. (2008). Secagem do Açafrão (Curcuma longa L.) em diferentes cortes geométricos. Cinética e Tecnologia de Alimentos, 28 (2), 387-394.

Wang, J., Law, Chung-Lim., Nema, P. K., Zhao, J-H., Liu, Z-L., Gao, Z-J., \& Xiao, H-W. (2018). Pulsed vacuum drying enhances drying kinetics and quality of lemon slices. Journal of Food Engineering, 224, 129-138. 